\title{
Stability of the Modified Craig-Sneyd scheme for two-dimensional convection-diffusion equations with mixed derivative term
}

\author{
K. J. in 't Hout* and C. Mishra ${ }^{\dagger}$
}

April 7, 2010

\begin{abstract}
The Modified Craig-Sneyd (MCS) scheme is a promising splitting scheme of the ADI type introduced by In 't Hout \& Welfert [Appl. Num. Math. 59 (2009)] for multi-dimensional pure diffusion equations having mixed spatial-derivative terms. In this paper we investigate the extension of the MCS scheme to two-dimensional convection-diffusion equations with a mixed derivative. Both necessary and sufficient conditions on the parameter $\theta$ of the scheme are derived concerning unconditional stability in the von Neumann sense.
\end{abstract}

Keywords: Initial-boundary value problems, convection-diffusion equations, method-of-lines, ADI splitting schemes, von Neumann stability analysis, Fourier transformation.

AMS subject classifications: 65L04, 65L05, 65L20, 65M12, 65M20, 91G60.

${ }^{*}$ Department of Mathematics and Computer Science, University of Antwerp, Middelheimlaan 1, 2020 Antwerp, Belgium (e-mail: karel.inthout@ua.ac.be).

$\dagger$ Department of Mathematics and Computer Science, University of Antwerp, Middelheimlaan 1, 2020 Antwerp, Belgium (e-mail: chittaranjan.mishra@ua.ac.be). 


\section{Introduction}

We consider the numerical solution of initial value problems for large systems of ordinary differential equations (ODEs),

$$
U^{\prime}(t)=F(t, U(t)) \quad(t \geq 0), \quad U(0)=U_{0},
$$

with given vector-valued function $F$, given initial vector $U_{0}$, and unknown vectors $U(t)$ (for $t>0$ ). Our interest in this paper lies in systems (1.1) that arise from semi-discretization of initial-boundary value problems for two-dimensional convection-diffusion equations possessing a mixed spatial-derivative term,

$$
\frac{\partial u}{\partial t}=d_{11} u_{x x}+\left(d_{12}+d_{21}\right) u_{x y}+d_{22} u_{y y}+c_{1} u_{x}+c_{2} u_{y} .
$$

Here $c=\left(c_{i}\right)$ and $D=\left(d_{i j}\right)$ denote a given real vector and a given positive semidefinite real matrix, respectively. A main application area of equations of the kind (1.2) is financial option pricing theory, where mixed derivative terms $u_{x y}$ arise naturally since the underlying Brownian motions are usually correlated to each other. Extensive details and examples of financial applications are given in, for example, the references [9, 10, 11].

For the numerical solution of semi-discrete problems (1.1), splitting schemes form an effective and popular means, cf. e.g. [5, 8]. This paper is devoted to the analysis of a recent splitting scheme of the Alternating Direction Implicit (ADI) type that has been tailored so as to deal with equations possessing a mixed derivative term. Let $\theta>0$ be a given fixed parameter. Assume the right-hand side function $F$ is decomposed into a sum

$$
F(t, v)=F_{0}(t, v)+F_{1}(t, v)+F_{2}(t, v),
$$

where $F_{0}$ represents the contribution to $F$ stemming from the mixed derivative term, and $F_{j}$ (for $j=1,2$ ) represents the contribution to $F$ stemming from all spatial derivative terms in the $j$-th spatial direction. Let $\Delta t>0$ be a given time step and define temporal grid points by $t_{n}=n \cdot \Delta t(n=0,1,2, \ldots)$. We consider the following splitting scheme for (1.1), generating in a one-step fashion successive approximations $U_{1}, U_{2}, U_{3}, \ldots$ to $U\left(t_{1}\right), U\left(t_{2}\right), U\left(t_{3}\right), \ldots$ :

$$
\left\{\begin{array}{l}
Y_{0}=U_{n-1}+\Delta t F\left(t_{n-1}, U_{n-1}\right) \\
Y_{j}=Y_{j-1}+\theta \Delta t\left(F_{j}\left(t_{n}, Y_{j}\right)-F_{j}\left(t_{n-1}, U_{n-1}\right)\right) \quad(j=1,2), \\
\widehat{Y}_{0}=Y_{0}+\theta \Delta t\left(F_{0}\left(t_{n}, Y_{2}\right)-F_{0}\left(t_{n-1}, U_{n-1}\right)\right), \\
\widetilde{Y}_{0}=\widehat{Y}_{0}+\left(\frac{1}{2}-\theta\right) \Delta t\left(F\left(t_{n}, Y_{2}\right)-F\left(t_{n-1}, U_{n-1}\right)\right) \\
\widetilde{Y}_{j}=\widetilde{Y}_{j-1}+\theta \Delta t\left(F_{j}\left(t_{n}, \widetilde{Y}_{j}\right)-F_{j}\left(t_{n-1}, U_{n-1}\right)\right) \quad(j=1,2), \\
U_{n}=\widetilde{Y}_{2}
\end{array}\right.
$$


Method (1.4) is called the Modified Craig-Sneyd (MCS) scheme. It has recently been introduced, in a slightly more general form, by In 't Hout \& Welfert [4]. Taylor expansion yields that the MCS scheme has classical order of consistency equal to two for any value $\theta$.

The MCS scheme can be viewed as an extension of the second-order CraigSneyd (CS) scheme proposed in 11. The latter scheme, called "iterated scheme" in loc. cit., is equivalent to (1.4) with parameter value $\theta=\frac{1}{2}$.

A perusal of (1.4) shows that the $F_{0}$ term is always treated explicitly, whereas the $F_{1}$ and $F_{2}$ terms are treated implicitly. More precisely, the MCS scheme starts with an explicit Euler step applied to the full system (1.1) which is succeeded by two implicit corrections corresponding to each of the two spatial directions. Subsequently, an explicit update is performed, which is followed again by two implicit, unidirectional corrections. Accordingly, the MCS scheme retains the well-known key advantage of ADI schemes over standard implicit methods, such as the Crank-Nicolson scheme, that the (linear or nonlinear) systems to be solved in each time step are much easier to handle.

The adaptation of ADI schemes to convection-diffusion equations with mixed derivative terms has been studied by a number of authors. Several stability results, in the sense of von Neumann, have been obtained. McKee et al. 6, 7. considered a simpler version of (1.4), which is equivalent to the first two lines with $U_{n}=Y_{2}$. This basic scheme, also known as the Douglas scheme, is of order one for any value $\theta$ in the presence of a mixed derivative term. McKee et al. showed that if $\theta=\frac{1}{2}$, then it is unconditionally stable when applied to a standard finite difference (FD) discretization of (1.2). Next, Craig \& Sneyd [1] formulated the second-order CS scheme and proved that this scheme is unconditionally stable in the case of (1.2) with $c \equiv \mathbf{0}$. Recently In 't Hout \& Welfert 3, 4, extended the above stability results in various ways. We state here the main results pertinent to the situation at hand. Firstly, for the CS scheme unconditional stability was proved [3] in the general case of (1.2). Secondly, it was shown [4] that in the case of (1.2) with $c \equiv \mathbf{0}$ the MCS scheme (1.4) is unconditionally stable whenever $\theta \geq \frac{1}{3}$.

Up to now it is an important open question when the MCS scheme, with $\theta \neq \frac{1}{2}$, is unconditionally stable in the application to general equations (1.2), i.e., with arbitrary $c$ and positive semi-definite $D$. As it turns out, an analysis of this is not straightforward, related to the fact that the eigenvalues of the semi-discrete linear operators move from the real line in the pure diffusion case to the complex plane in the general, convection-diffusion case. In the present paper we shall arrive at positive results on the above question.

For the stability analysis we consider the linear scalar test equation

$$
U^{\prime}(t)=\left(\lambda_{0}+\lambda_{1}+\lambda_{2}\right) U(t)
$$

with complex constants $\lambda_{j}(0 \leq j \leq 2)$. When applied to (1.5), the MCS scheme (1.4) reduces to the scalar iteration

$$
U_{n}=S_{\theta}\left(z_{0}, z_{1}, z_{2}\right) U_{n-1}
$$


with $z_{j}=\Delta t \cdot \lambda_{j} \quad(0 \leq j \leq 2)$ and

$$
S_{\theta}\left(z_{0}, z_{1}, z_{2}\right)=1+\frac{z_{0}+z}{p}+\theta \frac{z_{0}\left(z_{0}+z\right)}{p^{2}}+\left(\frac{1}{2}-\theta\right) \frac{\left(z_{0}+z\right)^{2}}{p^{2}},
$$

where we use the notation

$$
z=z_{1}+z_{2} \quad \text { and } \quad p=\left(1-\theta z_{1}\right)\left(1-\theta z_{2}\right) .
$$

The iteration (1.6) is stable if

$$
\left|S_{\theta}\left(z_{0}, z_{1}, z_{2}\right)\right| \leq 1
$$

In the von Neumann framework, the $\lambda_{j}$ represent eigenvalues of the linear operators $F_{j}$ that are obtained after semi-discretization, on a uniform spatial grid, of the convection-diffusion equation (1.2) with constant coefficients and periodic boundary condition. Corresponding to the positive semi-definiteness of the diffusion matrix $D$, it was shown in [3] (cf. also Sect. 3) that for standard FD discretizations the following condition on the scaled eigenvalues $z_{j}$ is fulfilled,

$$
\left|z_{0}\right| \leq 2 \sqrt{\Re z_{1} \Re z_{2}}, \quad \Re z_{1} \leq 0, \quad \Re z_{2} \leq 0
$$

where all bounds are sharp. In view of this, a natural stability requirement on the scheme (1.4) when applied to equations (1.2) with mixed derivative terms is that (1.8) holds whenever (1.9) is satisfied.

An outline of the rest of this paper is as follows. In Sect. 2 we study for which parameter values $\theta$ the implication (1.9) $\Rightarrow(1.8)$ is fulfilled. Four cases are investigated, depending on whether $z_{0}$ is real or complex valued and whether $z_{1}, z_{2}$ are (both) real or complex valued. In Sect. 3 the results of Sect. 2 are applied and discussed relevant to an application of the MCS scheme (1.4) to (1.2).

\section{Stability results for the MCS scheme}

Let $I$ denote the imaginary unit. In this section we study the stability requirement $(1.9) \Rightarrow(1.8)$. The following introductory result gives a criterion on $\theta$ for the case $z_{0}=0$. This is pertinent to the situation where no mixed derivative term is present in (1.2).

Theorem 2.1 There holds $\left|S_{\theta}\left(0, z_{1}, z_{2}\right)\right| \leq 1$ for all $z_{1}, z_{2} \in \mathbb{C}$ with $\Re z_{1} \leq 0$, $\Re z_{2} \leq 0$ if and only if $\theta \geq \frac{1}{4}$.

Proof The rational function $S_{\theta}\left(0, z_{1}, z_{2}\right)$ has no poles in the set $\Re z_{1}, \Re z_{2} \leq 0$ and therefore attains its maximum on the boundary of this set. Thus assume $z_{1}=I b_{1}, z_{2}=I b_{2}$ with $b_{1}, b_{2} \in \mathbb{R}$. We have

$$
\left|S_{\theta}\left(0, z_{1}, z_{2}\right)\right| \leq 1 \Longleftrightarrow\left|p^{2}+p z+\left(\frac{1}{2}-\theta\right) z^{2}\right|^{2}-\left|p^{2}\right|^{2} \leq 0 .
$$


Write $u=1-\theta^{2} b_{1} b_{2}$ and $v=b_{1}+b_{2}$. Then

$$
p=u-\theta I v, p^{2}=u^{2}-\theta^{2} v^{2}-2 \theta I u v, p z=\theta v^{2}+I u v, z^{2}=-v^{2}
$$

and it follows after some algebraic manipulations that

$$
\left|p^{2}+p z+\left(\frac{1}{2}-\theta\right) z^{2}\right|^{2}-\left|p^{2}\right|^{2}=\left[\left(\theta^{2}-2 \theta+\frac{1}{2}\right)^{2}-\theta^{4}\right] v^{4} .
$$

Hence,

$$
\left|S_{\theta}\left(0, z_{1}, z_{2}\right)\right| \leq 1 \quad \text { whenever } \Re z_{1}, \Re z_{2} \leq 0
$$

if and only if

$$
\left|\theta^{2}-2 \theta+\frac{1}{2}\right| \leq \theta^{2}
$$

which is equivalent to $\theta \geq \frac{1}{4}$.

In 4] the stability of ADI schemes for pure diffusion equations with mixed derivatives was analyzed. This concerns the case where all $z_{j}$ are real-valued. For the MCS scheme and two spatial dimensions, the following criterion on $\theta$ was obtained.

Theorem 2.2 There holds $\left|S_{\theta}\left(z_{0}, z_{1}, z_{2}\right)\right| \leq 1$ whenever $z_{0}, z_{1}, z_{2} \in \mathbb{R}$ satisfy (1.9) if and only if $\theta \geq \frac{1}{3}$.

Proof See [4, Thm. 2.5].

In most applications, also a convection term is present. Accordingly, one is led to considering complex-valued $z_{1}, z_{2}$. The next theorem gives a necessary condition on $\theta$ for this situation.

Theorem 2.3 Suppose $\left|S_{\theta}\left(z_{0}, z_{1}, z_{2}\right)\right| \leq 1$ for all $z_{0} \in \mathbb{R}$ and $z_{1}, z_{2} \in \mathbb{C}$ satisfying (1.9). Then $\theta \geq \frac{2}{5}$.

Proof The result is obtained by a Taylor expansion at the point $z_{0}=z_{1}=$ $z_{2}=0$. We take $z_{0}=-2 a$ and $z_{1}=z_{2}=a \eta$ where $\eta=1+I$ and $a \in \mathbb{R}$ with $a \uparrow 0$. This choice was found to be convenient after numerical experimentation. Inserting into (1.7) and using $1 /(1-\xi)=1+\xi+\xi^{2}+\mathcal{O}\left(\xi^{3}\right)(\xi \rightarrow 0)$, it follows that

$$
\begin{aligned}
S_{\theta}(-2 a, a \eta, a \eta) & =1+\frac{2 I a}{(1-\theta a \eta)^{2}}+\left(\theta-\frac{1}{2}-\theta I\right) \frac{4 a^{2}}{(1-\theta a \eta)^{4}} \\
& =1+2 I a-2 a^{2}+\left(20 \theta^{2}-8 \theta-8 \theta I\right) a^{3}+\mathcal{O}\left(a^{4}\right) .
\end{aligned}
$$

This yields

$$
\left|S_{\theta}(-2 a, a \eta, a \eta)\right|^{2}=1+\left(40 \theta^{2}-16 \theta\right) a^{3}+\mathcal{O}\left(a^{4}\right) .
$$

The right-hand side is bounded by 1 for $a \uparrow 0$ only if $40 \theta^{2}-16 \theta \geq 0$. Hence, it must hold that $\theta \geq \frac{2}{5}$. 
Based on strong numerical evidence (see Sect. 3) we conjecture that the condition on $\theta$ in Theorem 2.3 is also sufficient, but a proof is currently lacking.

The above results dealt with real-valued $z_{0}$. The two subsequent theorems concern arbitrary, complex-valued $z_{0}$. A preliminary result is

Lemma 2.4 Let $a, b, c \in \mathbb{R}$ be given. If $|a+b+c|=1$ and $\left|a \zeta^{2}+b \zeta+c\right| \leq 1$ whenever $\zeta \in \mathbb{C}$ with $|\zeta|=1$, then $a b+b c+4 a c \geq 0$.

Proof Consider the function $f$ defined by

$$
f(\varphi)=\left|a e^{2 I \varphi}+b e^{I \varphi}+c\right|^{2} \quad(\varphi \in \mathbb{R}) .
$$

There holds

$$
\begin{aligned}
f(\varphi) & =[a \cos (2 \varphi)+b \cos (\varphi)+c]^{2}+[a \sin (2 \varphi)+b \sin (\varphi)]^{2} \\
& =a^{2}+b^{2}+c^{2}+2 a b \cos (\varphi)+2 b c \cos (\varphi)+2 a c \cos (2 \varphi) .
\end{aligned}
$$

One readily verifies that $f(0)=1, f^{\prime}(0)=0, f^{\prime \prime}(0)=-2(a b+b c+4 a c)$ and hence

$$
f(\varphi)=1-(a b+b c+4 a c) \varphi^{2}+\mathcal{O}\left(\varphi^{3}\right) \quad(\varphi \rightarrow 0) .
$$

Using that $f(\varphi) \leq 1$ whenever $\varphi \in \mathbb{R}$, proves the assertion.

For the case where $z_{1}, z_{2}$ are real-valued, we obtain the following necessary lower bound on $\theta$. Numerical experiments indicate that this bound is sufficient as well.

Theorem 2.5 Suppose $\left|S_{\theta}\left(z_{0}, z_{1}, z_{2}\right)\right| \leq 1$ for all $z_{0} \in \mathbb{C}$ and $z_{1}, z_{2} \in \mathbb{R}$ satisfying (1.9). Then $\theta \geq \frac{5}{12}$.

Proof Setting $q=p^{2}+p z+\left(\frac{1}{2}-\theta\right) z^{2}$ and $w=p+(1-\theta) z$, we can write

$$
S_{\theta}\left(z_{0}, z_{1}, z_{2}\right)=\frac{\frac{1}{2} z_{0}^{2}+w z_{0}+q}{p^{2}} .
$$

Let $y=2 \sqrt{z_{1} z_{2}}$. Since $\left|S_{\theta}\left(z_{0}, z_{1}, z_{2}\right)\right| \leq 1$ for all $z_{0} \in \mathbb{C}$ with $\left|z_{0}\right| \leq y$ we have

$$
\left|\frac{1}{2} y^{2} \zeta^{2}+w y \zeta+q\right| \leq p^{2} \quad \text { for all } \zeta \in \mathbb{C} \text { with }|\zeta| \leq 1 .
$$

Assume $z_{1}=z_{2}$. Then $z=-y$ and it is easily seen that $\frac{1}{2} y^{2}+w y+q=p^{2}$. Therefore Lemma 2.4 can be applied and, using $y \geq 0$, this leads to the necessary condition

$$
\frac{1}{2} w y^{2}+q w+2 q y \geq 0 \quad \text { whenever } z_{1}=z_{2} \leq 0 .
$$

Denote $x=\theta y$. Then

$$
p=1+x+\frac{1}{4} x^{2} .
$$

Next, after some computations, there follows

$$
\frac{1}{2} w y^{2}+q w+2 q y=p^{3}+p^{2} x-\frac{1}{\theta}\left(x^{3}+2 p x^{2}\right) .
$$


By (2.11), we arrive at

$$
\theta \geq \frac{x^{3}+2 p x^{2}}{p^{3}+p^{2} x}
$$

The right-hand side is a rational function of $x \geq 0$, which is readily seen to have a global maximum at $x=2$. Inserting this value yields the lower bound $\theta \geq \frac{5}{12}$.

The final result in this section concerns the most general case, where all $z_{j}$ are complex-valued. To derive this result we employ a lemma from 3 pertinent to the condition (1.9). For completeness, its concise proof is included here.

Lemma 2.6 If $z_{1}, z_{2} \in \mathbb{C}$ with $\Re z_{1} \leq 0, \Re z_{2} \leq 0$, then

$$
2 \sqrt{\Re z_{1} \Re z_{2}} \leq\left|\frac{p}{2 \theta}\right|-\left|\frac{p}{2 \theta}+z\right| .
$$

Proof Define the vectors

$$
\mathbf{v}_{j}=\left(\begin{array}{c}
\sqrt{-2 \Re z_{j}} \\
\left|1+\theta z_{j}\right| / \sqrt{2 \theta}
\end{array}\right), \quad j=1,2 .
$$

Their Euclidean norms are

$$
\left\|\mathbf{v}_{j}\right\|=\sqrt{-2 \Re z_{j}+\frac{\left|1+\theta z_{j}\right|^{2}}{2 \theta}}=\frac{\left|1-\theta z_{j}\right|}{\sqrt{2 \theta}} .
$$

Next, their standard inner product is

$$
\left\langle\mathbf{v}_{1}, \mathbf{v}_{2}\right\rangle=2 \sqrt{\Re z_{1} \Re z_{2}}+\frac{\left|\left(1+\theta z_{1}\right)\left(1+\theta z_{2}\right)\right|}{2 \theta}=2 \sqrt{\Re z_{1} \Re z_{2}}+\left|\frac{p}{2 \theta}+z\right| .
$$

Applying the Cauchy-Schwarz inequality gives

$$
2 \sqrt{\Re z_{1} \Re z_{2}}+\left|\frac{p}{2 \theta}+z\right| \leq \frac{\left|1-\theta z_{1}\right|\left|1-\theta z_{2}\right|}{2 \theta}=\left|\frac{p}{2 \theta}\right|,
$$

which concludes the proof.

For the most general case, we have the following positive result:

Theorem 2.7 If $\frac{1}{2} \leq \theta \leq 1$, then $\left|S_{\theta}\left(z_{0}, z_{1}, z_{2}\right)\right| \leq 1$ whenever $z_{0}, z_{1}, z_{2} \in \mathbb{C}$ satisfy (1.9).

Proof The expression (2.10) for $S_{\theta}$ yields

$$
\left|S_{\theta}\left(z_{0}, z_{1}, z_{2}\right)\right| \leq \frac{1}{2}\left|\frac{z_{0}}{p}\right|^{2}+\left|\frac{z_{0}}{p}\right|\left|1+(1-\theta) \frac{z}{p}\right|+\left|1+\frac{z}{p}+\left(\frac{1}{2}-\theta\right) \frac{z^{2}}{p^{2}}\right| .
$$

By invoking Lemma 2.6 it follows that $\left|S_{\theta}\left(z_{0}, z_{1}, z_{2}\right)\right|$ is bounded from above by

$$
\frac{1}{2}\left(\frac{1}{2 \theta}-\left|\frac{1}{2 \theta}+\frac{z}{p}\right|\right)^{2}+\left(\frac{1}{2 \theta}-\left|\frac{1}{2 \theta}+\frac{z}{p}\right|\right)\left|1+(1-\theta) \frac{z}{p}\right|+\left|1+\frac{z}{p}+\left(\frac{1}{2}-\theta\right) \frac{z^{2}}{p^{2}}\right| .
$$


We can write

$$
1+2 \theta \frac{z}{p}=r e^{I \varphi} \quad \text { with } 0 \leq r \leq 1 \text { and } 0 \leq \varphi<2 \pi .
$$

Define

$$
\begin{aligned}
& f_{1}(\varphi, r)=\left|2 \theta+(1-\theta)\left(r e^{I \varphi}-1\right)\right|, \\
& f_{2}(\varphi, r)=\left|8 \theta^{2}+4 \theta\left(r e^{I \varphi}-1\right)+(1-2 \theta)\left(r e^{I \varphi}-1\right)^{2}\right| .
\end{aligned}
$$

Then it follows that

$$
\left|S_{\theta}\left(z_{0}, z_{1}, z_{2}\right)\right| \leq \frac{(1-r)^{2}+2(1-r) f_{1}(\varphi, r)+f_{2}(\varphi, r)}{8 \theta^{2}} .
$$

Let $\frac{1}{2} \leq \theta \leq 1$. We prove that the right-hand side of (2.12) is bounded by 1 for all $0 \leq r \leq 1,0 \leq \varphi<2 \pi$. First note that $f_{j}(2 \pi-\varphi, r)=f_{j}(\varphi, r)$ $(j=1,2)$ and therefore it suffices to consider $0 \leq \varphi \leq \pi$. Let $r \in[0,1]$ be fixed but arbitrary and define $g_{j}(\varphi)=f_{j}(\varphi, r)^{2}(j=1,2)$. For the function $g_{1}$ it is readily verified that

$$
g_{1}^{\prime}(\varphi)=-2(3 \theta-1)(1-\theta) r \sin \varphi .
$$

This directly implies that $g_{1}$, and hence $f_{1}$, is nonincreasing on $[0, \pi]$. For the function $g_{2}$ a more elaborate computation shows

$$
g_{2}^{\prime}(\varphi)=4(2 \theta-1)(4 \theta-1)\left[2(2 \theta-1) r \cos \varphi+r^{2}-(4 \theta-1)\right] r \sin \varphi .
$$

In view of

$$
2(2 \theta-1) r \cos \varphi+r^{2}-(4 \theta-1) \leq 2(2 \theta-1)+1-(4 \theta-1)=0
$$

we find that also $g_{2}$, and hence $f_{2}$, is nonincreasing on $[0, \pi]$. Consequently, it is sufficient to prove that the right-hand side of (2.12) is bounded by 1 whenever $0 \leq r \leq 1, \varphi=0$. Write $s=r-1 \in[-1,0]$. One easily verifies that

$$
\begin{aligned}
& f_{1}(0, r)=2 \theta+(1-\theta) s, \\
& f_{2}(0, r)=8 \theta^{2}+4 \theta s+(1-2 \theta) s^{2} .
\end{aligned}
$$

Inserting this and rearranging terms, it follows that the upper bound (2.12) is (in fact) equal to 1 whenever $0 \leq r \leq 1, \varphi=0$.

Numerical evidence leads to the conjecture that the conclusion of Theorem 2.7 is valid for all $\theta \geq \frac{5}{12}$, i.e., under the (necessary) lower bound of Theorem 2.5 . A proof of this does not appear to be straightforward. We note that in the proof above the assumption $\frac{1}{2} \leq \theta \leq 1$ is used in an essential manner. 


\section{Application and discussion}

In this section we discuss an application to convection-diffusion equations (1.2). We semi-discretize on the unit square $[0,1] \times[0,1]$ by using central second-order FD schemes on a Cartesian grid with mesh widths $\Delta x$ and $\Delta y$ in the $x$ and $y$ directions, respectively:

$$
\begin{aligned}
\left(u_{x}\right)_{i, j} \approx & \frac{u_{i+1, j}-u_{i-1, j}}{2 \Delta x} \\
\left(u_{y}\right)_{i, j} \approx & \frac{u_{i, j+1}-u_{i, j-1}}{2 \Delta y} \\
\left(u_{x x}\right)_{i, j} \approx & \frac{u_{i+1, j}-2 u_{i, j}+u_{i-1, j}}{(\Delta x)^{2}} \\
\left(u_{y y}\right)_{i, j} \approx & \frac{u_{i, j+1}-2 u_{i, j}+u_{i, j-1}}{(\Delta y)^{2}} \\
\left(u_{x y}\right)_{i, j} \approx & \frac{(1+\beta)\left(u_{i+1, j+1}+u_{i-1, j-1}\right)-(1-\beta)\left(u_{i-1, j+1}+u_{i+1, j-1}\right)}{4 \Delta x \Delta y} \\
& +\frac{4 \beta u_{i, j}-2 \beta\left(u_{i+1, j}+u_{i, j+1}+u_{i-1, j}+u_{i, j-1}\right)}{4 \Delta x \Delta y}
\end{aligned}
$$

Here $\beta$ denotes a real parameter with $-1 \leq \beta \leq 1$ and we use the notation $u_{i, j}=u(i \Delta x, j \Delta y, t)$. We note that the right-hand side of (3.1p) is the most general form of a second-order FD approximation of the mixed derivative $u_{x y}$ based on a centered 9-point stencil. When $\beta=0$, it reduces to the well-known 4 -point formula

$$
\left(u_{x y}\right)_{i, j} \approx \frac{u_{i+1, j+1}+u_{i-1, j-1}-u_{i-1, j+1}-u_{i+1, j-1}}{4 \Delta x \Delta y} .
$$

Assuming constant coefficients and a periodic boundary condition for (1.2), the above FD discretization yields a splitted, semi-discrete system (1.1), (1.3) where $F_{j}(t, v)=A_{j} v$ for $j=0,1,2$ with constant matrices $A_{j}$. The matrix $A_{0}$ represents the cross derivative term in (1.2) and $A_{1}, A_{2}$ represent the spatial derivatives in the $x$ and $y$ directions, respectively. The periodicity condition implies that the $A_{j}$ are Kronecker products of circulant (thus normal) matrices that commute with each other, and are therefore simultaneously diagonalizable by a unitary matrix. Hence, stability can be rigorously analyzed by considering the scalar test equation (1.5) with $\lambda_{j}$ eigenvalues of $A_{j}(0 \leq j \leq 2)$. This is equivalent to a von Neumann stability analysis. By inserting discrete Fourier modes, it follows that the scaled eigenvalues $z_{j}$ are given by

$$
\begin{aligned}
& z_{0}=\left(d_{12}+d_{21}\right) b\left[-\sin \phi_{1} \sin \phi_{2}+\beta\left(1-\cos \phi_{1}\right)\left(1-\cos \phi_{2}\right)\right] \\
& z_{1}=-2 d_{11} a_{1}\left(1-\cos \phi_{1}\right)+I c_{1} q_{1} \sin \phi_{1}, \\
& z_{2}=-2 d_{22} a_{2}\left(1-\cos \phi_{2}\right)+I c_{2} q_{2} \sin \phi_{2},
\end{aligned}
$$

where

$$
a_{1}=\frac{\Delta t}{(\Delta x)^{2}}, \quad a_{2}=\frac{\Delta t}{(\Delta y)^{2}}, \quad b=\frac{\Delta t}{\Delta x \Delta y}, \quad q_{1}=\frac{\Delta t}{\Delta x}, \quad q_{2}=\frac{\Delta t}{\Delta y} .
$$


The angles $\phi_{j}$ are integer multiples of $2 \pi / m_{j}(j=1,2)$ where $m_{1}, m_{2}$ are the dimensions of the grid in the $x$ and $y$ directions, respectively.

Using the positive semi-definiteness of the diffusion matrix $D$, an elementary calculation shows $\left[3\right.$ that $z_{0}, z_{1}, z_{2}$ fulfill the condition (1.9), independently of $\Delta t, \Delta x, \Delta y$. Upon invoking Theorem 2.7 the following neat stability result is obtained for the MCS scheme applied to (1.2).

Theorem 3.1 Consider equation (1.2) with positive semi-definite matrix $D$ and periodic boundary condition. Let the semi-discrete system (1.1), (1.3) be obtained after FD discretization and splitting as described in this section. Then the MCS scheme (1.4) is unconditionally stable when applied to (1.1), (1.3) whenever $\frac{1}{2} \leq \theta \leq 1$. Moreover, this conclusion remains valid when any other stable FD discretizations for $u_{x}, u_{y}$ are used in place of (3.1 a), (3.1b).

The last part of Theorem 3.1 follows directly from the fact that the real parts of the new eigenvalues $z_{1}, z_{2}$ are always smaller than those of (3.2 b), (3.2. $)$, respectively, and hence, (1.9) remains true.

An inspection of $(3.2 \mathrm{k})$ yields that the eigenvalues $z_{0}$ have the property that their imaginary part is identically equal to zero. Accordingly, it is of particular interest to know all parameter values $\theta$ such that the stability requirement $(1.9) \Rightarrow(1.8)$ holds for just real-valued $z_{0}$. Theorem 2.7 provides the sufficient condition $\frac{1}{2} \leq \theta \leq 1$, whereas Theorem 2.3 yields the necessary condition $\theta \geq \frac{2}{5}$.

Next, we remark that the MCS scheme has recently been applied successfully in 22 to actual convection-diffusion equations (1.2) with mixed derivative terms using the parameter value $\theta=\frac{1}{3}$. This seems to be surprising, as this value was determined 4 for pure diffusion equations (1.2) and it clearly does not satisfy the necessary condition $\theta \geq \frac{2}{5}$ for equations with convection. We note that reasons for choosing a smaller $\theta$ in the MCS scheme are a reduced error constant and better damping properties compared to the original CS scheme, see [2].

Theoretical results on the latter two issues are not known at this moment. To gain insight, we have performed a numerical experiment. Let $r_{1,0}$ and $r_{i, j}$ for $i, j=1,2$ denote independent, uniformly distributed random numbers in $[0,1]$ and consider random triplets $\left(z_{0}, z_{1}, z_{2}\right)$ given by

$$
z_{0}=\left(2 r_{1,0}-1\right) \cdot 2 \sqrt{\Re z_{1} \Re z_{2}} \quad \text { and } \quad z_{j}=-10^{1-5 r_{1, j}} \pm I 10^{1-5 r_{2, j}} \quad(j=1,2) .
$$

Then (1.9) holds and $z_{0} \in \mathbb{R}$. For each $\theta=\frac{1}{4}+\frac{k}{400}$ with $k=0,1, \ldots, 100$ we computed the maximum value of $\left|S_{\theta}\left(z_{0}, z_{1}, z_{2}\right)\right|$ over two million points $\left(z_{0}, z_{1}, z_{2}\right)$ above. The outcome is displayed in Figure 1 .

Figure 1 reveals the intriguing result that the estimated maximum value of $\left|S_{\theta}\right|$ is very close to 1 whenever $\theta \geq \frac{1}{3}$. For $\theta=\frac{1}{3}$ we arrive at a maximum value of 1.02. Additional experiments in this case suggest that $\left|S_{\theta}\right|$ is larger than 1 for a limited set of points $\left(z_{0}, z_{1}, z_{2}\right)$, and at most 1 under only a slightly stronger condition on $z_{0}$ than in (1.9). Because of these observations, it is very plausible that the MCS scheme performs well in actual applications to (1.2), also with convection, already when $\theta=\frac{1}{3}$. 


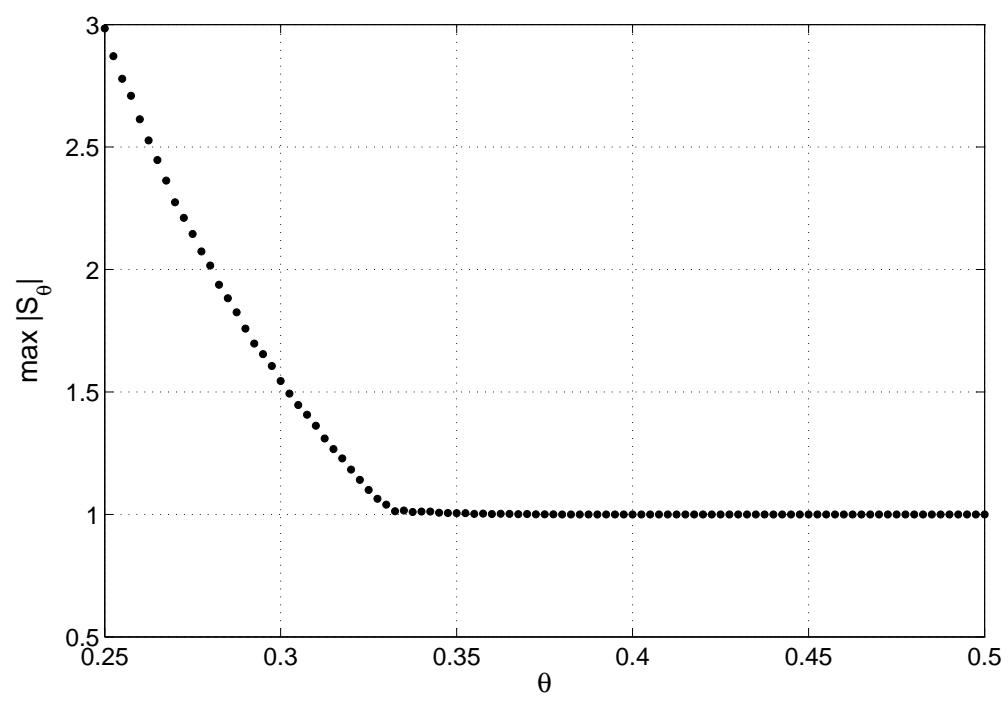

Figure 1: Estimated maximum of $\left|S_{\theta}\left(z_{0}, z_{1}, z_{2}\right)\right|$ under (1.9) with $z_{0} \in \mathbb{R}$.

Subsequently, an examination of the obtained numerical results indicates that $\left|S_{\theta}\right| \leq 1$ for all $\theta \geq \frac{2}{5}$. This supports our conjecture formulated below Theorem 2.3 .

In view of the above, it is likely that the condition on $\theta$ in Theorem 3.1 can be relaxed to $\theta \geq \frac{2}{5}$, and next, that a slightly modified version of Theorem 3.1 holds under the (weaker) assumption $\theta \geq \frac{1}{3}$. In future research we intend to study these issues theoretically.

\section{Acknowledgments}

This work has been supported financially by the Research Foundation-Flanders, FWO contract no. G.0125.08.

\section{References}

[1] I. J. D. Craig \& A. D. Sneyd, An alternating-direction implicit scheme for parabolic equations with mixed derivatives, Comp. Math. Appl. 16 (1988) 341-350.

[2] K. J. in 't Hout \& S. Foulon, ADI finite difference schemes for option pricing in the Heston model with correlation, Int. J. Num. Anal. Mod. 7 (2010) 303-320. 
[3] K. J. in 't Hout \& B. D. Welfert, Stability of ADI schemes applied to convection-diffusion equations with mixed derivative terms, Appl. Num. Math. 57 (2007) 19-35.

[4] K. J. in 't Hout \& B. D. Welfert, Unconditional stability of second-order ADI schemes applied to multi-dimensional diffusion equations with mixed derivative terms, Appl. Num. Math. 59 (2009) 677-692.

[5] W. Hundsdorfer \& J. G. Verwer, Numerical Solution of Time-Dependent Advection-Diffusion-Reaction Equations, Springer, Berlin, 2003.

[6] S. McKee \& A. R. Mitchell, Alternating direction methods for parabolic equations in two space dimensions with a mixed derivative, Comp. J. 13 (1970) 81-86.

[7] S. McKee, D. P. Wall \& S. K. Wilson, An alternating direction implicit scheme for parabolic equations with mixed derivative and convective terms, J. Comp. Phys. 126 (1996) 64-76.

[8] A. R. Mitchell \& D. F. Griffiths, The Finite Difference Method in Partial Differential Equations, Wiley, Chichester, 1980.

[9] S. E. Shreve, Stochastic Calculus for Finance II, Springer, New York, 2004.

[10] D. Tavella \& C. Randall, Pricing Financial Instruments, Wiley, New York, 2000.

[11] P. Wilmott, Derivatives. Wiley, Chichester, 1999. 